\title{
Prevalence of Onchocerciasis Infection in the Sub-Saharan Africa Countries: A Systematic Review and Meta-analysis
}

\author{
Mitiku Bonsa Debela ${ }^{1, *}$, Dejene Hailu Kassa2 ${ }^{2}$ Taklu Marama Mokonnon ${ }^{3}$
}

\section{Mitiku Bonsa Debela ${ }^{1, *}$, Dejene Hailu Kassa ${ }^{2}$, Taklu Marama Mokonnon ${ }^{3}$}

'Department of Public Health, School of Medicine and Health Sciences, Madda Walabu University, ETHIOPIA.

${ }^{2}$ Schools of Public Health, College of Medicine and Health Sciences, Hawassa University, ETHIOPIA.

${ }^{3}$ Schools of Midwifery, College of Health Sciences and Medicine, Wolaita Sodo University, ETHIOPIA.

\section{Correspondence}

Mr. Mitiku Bonsa Debela

Department of Public Health, School of Medicine and Health Sciences, Madda Walabu University, ETHIOPIA.

Phone no: +251967776951

Email: mitikubonsa8@gmail.com

\section{History}

- Submission Date: 18-05-2021

- Revised Date: 24-06-2021

- Accepted Date: 19-07-2021

DOI : 10.5530/ijmedph.2021.4.32

\section{Article Available online}

http://www.jjmedph.org/v11/i4

\section{Copyright}

(C) 2021 Phcog.Net. This is an openaccess article distributed under the terms of the Creative Commons Attribution 4.0 International license.

\begin{abstract}
Background: Findings from many African countries show that the prevalence of onchocerciasis infection is fragmented and in a wide range. Clear and organized evidence that showed the prevalence is limited. Therefore, this systematic review and meta-analysis estimated the pooled prevalence of onchocerciasis infection in the sub-Saharan African Countries. Methods: Published articles found in Scopus, PubMed/Medline, Science Direct, Google Scholar, and Cochrane Library databases were systematically searched. Based on the recommendations of the Preferred Reporting Items for Systematic Reviews and Meta-Analyses (PRISMA) was employed to determine the prevalence for onchocerciasis infection among peoples of all age groups in Africa. Observational studies from 1 January 2015 to 10 February 2021 revealed the prevalence of onchocerciasis in the sub-Saharan African countries was incorporated. The pooled prevalence of the studies was computed using STATA version 14 statistical software. The heterogeneity of the study was assessed using Cochrane $\mathrm{Q}$ test statistics, the I-squared values test, and the Galbraith plot. Considering within and between variability, the randomeffect model was used to determine the pooled prevalence. Funnel plot and egger's tests were conducted to assess publication bias. Results: Out of 1985 accessed studies, 17 studies fulfilled the eligibility criteria and were included to estimate the pooled prevalence of onchocerciasis infection. The pooled prevalence of onchocerciasis was 30\% (95\% Cl: 13,47) in sub-Saharan Africa. Thirty-two percent (95\% Cl: 9, 56)) and $28 \%(95 \% \mathrm{Cl}: 2,54)$ of onchocerciasis infection were identified from the study conducted before 2015 and after 2015, respectively, based on the subgroup analysis. The prevalence of onchocerciasis among farmers and housewives was 35\% (95\% Cl: 12, 58), 18\% (95\% Cl: 10, 27), respectively. Conclusion: Onchocerciasis is still of immense public health importance. Hence, the local government and other stakeholders should implement rigorous and comprehensive onchocerciasis prevention strategies such as improved sanitation, vector control, mass drug administration campaigns, and multifaceted methods based on their context.

Key words: Onchocerciasis, Prevalence, Meta-analysis, Systematic review, Africa.
\end{abstract}

\section{INTRODUCTION}

Neglected tropical diseases (NTDs) are a category of infections caused by bacteria, parasites, viruses, and fungi. Many tropical and sub-tropical developing countries, where poverty is widespread, have them. NTDs impact over 1.5 billion people in the world's poorest, most disadvantaged and isolated populations. ${ }^{1,2}$ The five most common NTDs in the world are lymphatic filariasis, onchocerciasis, Schistosomiasis, soil-transmitted helminthes, and trachoma, all of which are commonly treated with mass drug administration's. ${ }^{3,4}$ The parasitic disease onchocerciasis (river blindness) is caused by the filarial worm onchocercal volvulus, which is transmitted by blackflies. Extreme itching, skin patches, and vision damage, including blindness, are all symptoms of the disorder. It is one of eleven NTDs that the World Health Organization (WHO) has recently targeted for eradication. ${ }^{5,6}$

The WHO reports that 198 million people are at risk of contracting onchocerciasis, but this number could

rise as the mapping of low-transmission areas is completed. ${ }^{7}$ In 2013, the global burden of disease (GBD) report reported that 17 million people were infected worldwide. ${ }^{8}$ With 8.3 million cases, the Democratic Republic of Congo had the largest number of onchocerciasis cases. ${ }^{9}$ The GBD collaborators reported an overall prevalence of 15.53 million in 2015, with 12.22 million cases of skin disease and 1.03 million cases of onchocerciasis-related vision loss. According to the most recent available data from the GBD report 2016, the global prevalence is estimated to be 14.65 million. $^{10}$

Furthermore, in endemic regions, mostly in SubSaharan Africa, an estimated 90 million people are at risk of contracting the disease, with more than 37 million infected and 300,000 permanently blind as a result of onchocerciasis. ${ }^{11}$ In 2019, the World Health Organization (WHO) reported that 217.5 million people worldwide need mass ivermectin administration. ${ }^{12}$ 
Onchocerciasis is becoming less of a public health issue globally, and it is no longer a concern in some areas. ${ }^{13}$ Despite the fact that the prevalence of onchocerciasis has decreased significantly in African countries, the disease remains a major public health concern. Onchocerciasis is a parasitic disease that affects about 37 million people in 34 countries, with the majority of cases occurring in Africa, with a few isolated cases in South and Central America. ${ }^{14}$

In addition, It is estimated that 86 million people live in high risk areas in the African programme for onchocerciasis control (APOC) countries. ${ }^{15}$ Finding from Sierra Leone indicates that onchocerciasis is hyperendemic in the Georama Chiefdom. Of the 651 people examined, 472 (72.5\%) had one or more microfilaria of onchocercal volvulus. ${ }^{16}$ In Congo 28 confirmed onchocerciasis infection was reported. ${ }^{17}$ In Tanzania, the prevalence of onchocercal skin symptoms were found in $170(38.8 \%)$, of which $30(6.9 \%)$ had nodules, $48(11.0 \%)$ chronic onchodermatitis and $92(21 \%)$ itching and the overall prevalence of onchocercal volvulus positive skin snips at baseline was $49 \%{ }^{18,19}$ In Yemen onchocerciasis is one of the most neglected diseases making the prevalence of $18.5 \%{ }^{20}$

In conclusion, the findings from various of the African continent is fragmented, not informative and a wide range of prevalence of onchocerciasis infection: they were reported ranging from $6.32 \%$ to $17 \%$ in Ethiopia. ${ }^{21-23} 2.43 \%$ to 13.2 in Ghana, ${ }^{24} 1.7 \%$ to $23.7 \%$ in Uganda, ${ }^{25} 7 \%$ to $19 \%$ in Cameron, ${ }^{26-28} 5 \%$ to $73 \%$ in Côte d'Ivoire and Burkina Faso, ${ }^{29} 49 \%$ to $83.2 \%$ in Tanzania. ${ }^{30}$

Therefore, understanding the pooled prevalence of onchocerciasis infection is paramount to design sound preventive and control strategies targeted to eliminate and eradicate the newly appearing infection in the region. Besides, as described above, the Sub-Saharan Africa countries are highly affected by neglected tropical diseases, which are projected to be exacerbated by low economic settings. Consequently, understanding what research has been conducted and what knowledge gaps remain regarding onchocerciasis infection is crucial to informing public health interventions.

Hence, the purpose of this systematic review and meta-analysis was aimed to estimate the pooled prevalence of onchocerciasis infection among peoples of all age groups in sub-Saharan Africa countries and to provide the necessary information to the scientific communities and policymakers who intervene in the problem.

\section{MATERIALS AND METHODS}

\section{Study design and setting}

This systematic review and meta-analysis will be conducted in subSaharan Africa from October 1/2020 to February 10/2021. Sub-Saharan Africa is the African continent that lies south of the Sahara that includes; Angola, Benin, Botswana, Burkina Faso, Burundi, Cameroon, Central African Republic, Chad, Congo, Cote d'Ivoire, Eritrea, Ethiopia, Gabon, Gambia, Ghana, Guinea, Guinea-Bissau, Kenya, Lesotho, Liberia, Madagascar, Malawi, Mali, Mauritania, Mauritius, Mozambique, Namibia, Niger, Nigeria, Rwanda, Senegal, Sierra Leone, Somalia, South Africa, United Republic of Tanzania, Togo, Uganda, Zaire, Zambia, Zimbabwe. ${ }^{31}$ Moreover, Sub-Saharan Africa had a population of 1.1 billion making the current rate of growth is $2.3 \%$. For the area, the UN predicts a population of between 2 and 2.5 billion by 2050, with a population density of 80 per square kilometer. More than $40 \%$ of the population is younger than 15 years old. ${ }^{32-34}$ With nearly $11 \%$ of the world's population bearing more than $24 \%$ of the global burden of disease, Sub-Saharan Africa is home to just $3 \%$ of the global health workers and invests less than $1 \%$ of the world's financial capital on health. Health workers are concentrated in major cities and towns in most developed countries. Simultaneously, rural areas can only boast about $23 \%$ and $38 \%$ of doctors and nurses in the country, respectively. Although targets for sustainable development include at least 4.45 trained health professionals per 1,000 population, many sub-Saharan Africa regions fell below the WHO guideline in which the minimum threshold level for greater accessibility of critical services and to minimize the risk of infection as a result of work overload is 2.3 health workers per 1000 population. ${ }^{35}$

\section{Searching strategies}

First, search was done on the Cochrane Library, Joanna Briggs Institute (JBI), and PROSPERO databases to check whether a systematic review and meta-analysis studies exist or for the presence of ongoing review projects related to prevalence of onchocerciasis in sub-Saharan Africa. The review process followed the PRISMA guidelines to show accessed, screened, rejected and included articles systematically or as per predesigned searching strategies. Articles were accessed from SCOPUS, PubMed, Science Direct, Cochrane Library databases, Google Scholar (search engine) and African journals online. Grey literature like surveillance reports, academic dissertations, and conference abstracts was also be examined and included when it fulfills the inclusion criteria.

For this review, relevant articles were identified using the following Mesh terms. PubMed search strategy; (Prevalence) OR (magnitude) OR (Burden) OR (epidemiology) AND (onchocerciasis) OR (river blindness) OR (onchocerciasis volvulus) OR (onchocerciasis infection) OR (microfilaria) AND sub-Saharan Africa. The key terms were used in combination using Boolean operators like "OR" or "AND." The review restricted to full texts, free articles, and English language publications. This search involved articles published from 1 January 2015 to 10 February 2021. Besides, during the advanced PubMed search, it was used all fields and Mesh words. The first reviewer was performing the initial search and completes it on $10 / 02 / 2021$. The review was then scanning the literature for updates.

\section{Eligibility of the study Inclusion criteria}

This systematic review and meta-analysis considered all researches conducted in African countries that have identified the prevalence of onchocerciasis fulfilling the following elements:

Study design; Observational study design.

Time frame: All studies published from 1 January 2015 up to 10 February 2021

Publication type: Both published and unpublished studies.

Language: An article published in English language was included.

Study area: Studies conducted in Africa, which are methodologically institutional-based.

Population: All people of age groups.

Outcome: Studies that reported the outcome of interest (onchocerciasis infection)

\section{Exclusion criteria}

Those articles not entirely accessed during the time of searching process were omitted after attempted at least twice with the principal investigator via email. After reviewing their full texts, studies that did not report outcome of interest and with methodological problems were removed. Besides, studies with low quality as pre-settled parameters and review papers were also omitted. The full-text review was limited to studies that have met the requirements for inclusion.

\section{Quality assessment}

The database search results were merged, and duplicate papers were removed using Endnote (version X8). To assess the methodological 
qualities of the included articles, a modified version of the NewcastleOttawa quality assessment tool scale for cross-sectional studies was adapted and used to assess each study's quality. Three independent reviewers were critically appraising each paper. Disagreements were resolved by discussion among those reviewers. To address contradictions among the three independent reviewers, another reviewer was involved by taking the three authors' mean score or applying the third author. The original studies, which scored $\geq 7$ out of 10 , were considered high quality and included in the final meta-analysis. The three authors (MB, TM, and GR) were then independently assessing the quality of included research articles using the above tool.

\section{Data extraction}

Data were extracted using a structured data extraction spreadsheet (Microsoft excels). The corresponding author of the original research was contacted for additional information or to clarify method details as needed. All the abstracts included during the title and abstract review go to full-text review, and the necessary data were extracted using the prepared spreadsheet. Data was defined and extracted by MB and double-checked by a second reviewer in a pilot excel sheet. Authors were notified if the data for selecting papers are incomplete or ambiguous. Besides, two writers ( $\mathrm{MB}$ and $\mathrm{TM}$ ) independently extracted all the required data using Microsoft Excel. The outcome of interest data extraction format (prevalence) consists of the first author's name, publication year, and study location, the design of the analysis, sample size, number of participants with the outcome (case), occupation, gender, resident, and response rate.

\section{Outcome of measurement}

After identification, the PROSPERO registration number was CRD42021245110. The findings of the researches were reported in two ways; the prevalence of onchocerciasis as percentage or as the number of cases (n)/total number of participants in the sample $(\mathrm{N})$. These two parameters were important in the meta-analysis to estimate the pooled prevalence of onchocerciasis infection. Therefore, the prevalence rate was determined by dividing the number of individuals infected by the total number of participants in the study (sample size) multiplied by 100 .

\section{Data analysis}

The extracted data was imported into STATA 14 version software for analysis. Meta-analytic integration was carried out using STATA 14 version software and its "Metaprop" and "galbr" commands and the individual study prevalence estimations. The' Metaprop' command was explicitly developed for proportion meta-analysis and was based on the double arcsine transformation of Freeman-Tukey for stabilizing variances. Using Der Simonian and Laird random-effects models, systematic review was computed with Metaprop, a Stata command for pooling proportions, and presented in a forest plot with corresponding 95\% CIs. Publication bias was checked by funnel plot using the "metafunnel" command and by Egger's and Begg's test. Symmetrical graph was interpreted based on the graph's shape to indicate the lack of publication bias. In contrast, an asymmetrical graph was interpreted to indicate the presence of publication bias. Both Egger's and Begg's test was used as a cutoff point to declare the existence of publication bias with a $p$-value of less than 0.05 . To visualize the existence of heterogeneity, we were subjectively using Galbraith plot and Forest plot. Also, objectively (statistical test) using Higgins I-Squared $\left(\mathrm{I}^{2}\right)$, and Cochran's $\mathrm{Q}$ statistic was used. I-square statistics was quantifying the impact of heterogeneity on the meta-analysis across studies, and was a cutoff point of $50 \%$ was used to declare significant/ considerable heterogeneity.
By subgroup analysis, meta-regression, and sensitivity analysis the possible variations between the studies were discussed. Subgroup analysis was done based on the type of occupation, sample size, and year of publication to Figure out the possible source of heterogeneity across the studies. The results were presented using a random-effects meta-analysis model via forest plot with the corresponding odds ratio and 95\% confidence intervals.

The prevalence rate, the logarithm of prevalence, and standard error (SE) of the logarithm of prevalence was computed. The pooled prevalence of onchocerciasis with a $95 \%$ confidence interval was computed using random-effects model. To estimate the pooled effect size, random effect model was used to account within and between study variability. Due to the limited number, non-linear logistic regression analysis was used after extending studies into unit record archives. An output in meta-analyses was double-checked for internal consistency by the same person.

\section{RESULTS}

\section{Selection and identification of studies}

A total of 1985 papers were accessed from PubMed databases $(n=900)$, SCOPUS ( $n=145)$, Google scholar (search engine) $(n=600)$, and manual search, including gray literature $(n=80$ articles) and science direct $(n=260)$. From the total accessed papers, 987 studies have been omitted due to duplication. After reading the title and the abstract, 956 studies were omitted because they were not in line with this review's purpose and methodological deficit. Finally, 332 studies were screened for full-text review, 17 studies were included for this systematic review and meta-analysis (Figure 1).

\section{Characteristics of included studies}

A total of 17 primary studies with a total sample size of 17,756 were included in the review. In addition, the response rate in each included study was evaluated, and it was found that the response rate ranging from $83-100 \%$, and almost all the studies had a good response rate having a response rate of above $80 \%$. All of 17 reviewed studies were published in reputable journals were cross-sectional concerning the research design. ${ }^{36-51}$ Finally, the quality score of the studies ranges from 7-9 out of 10 points (Table 1 ).

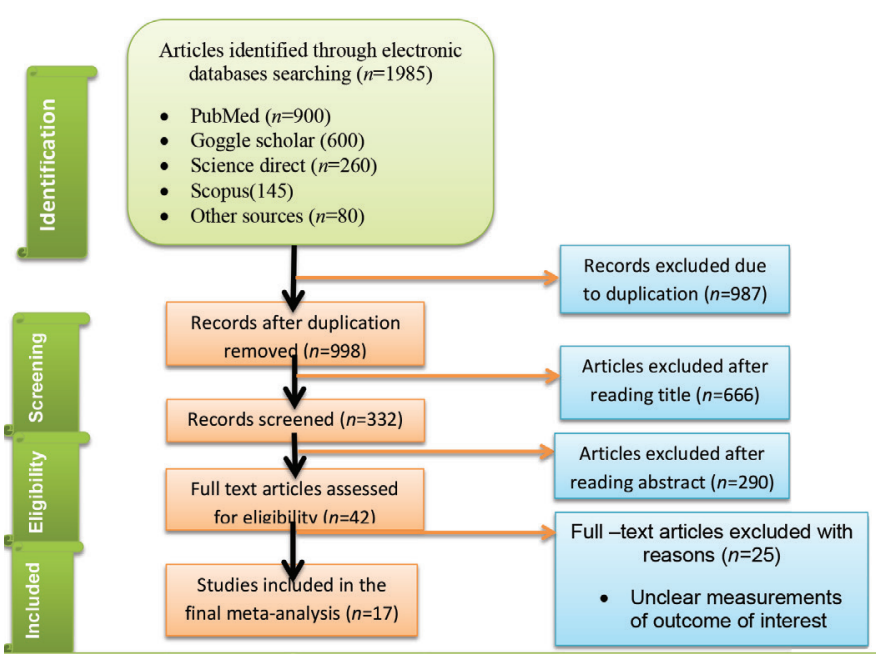

Figure 1: PRISMA flow diagram for study selection for systematic review and meta-analysis of prevalence onchocerciasis in sub-Saharan Africa, 2021 $(n=17)$. 


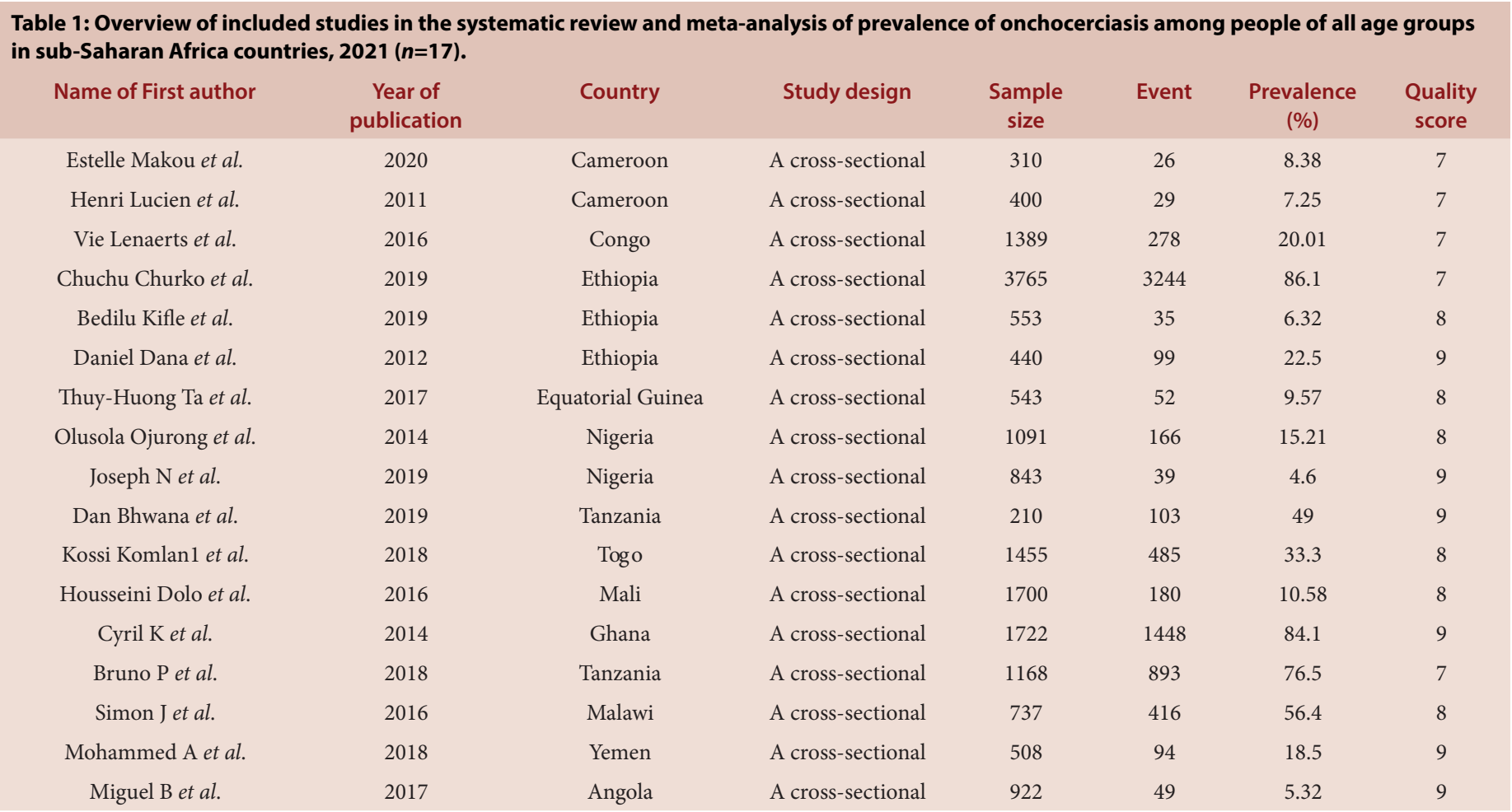

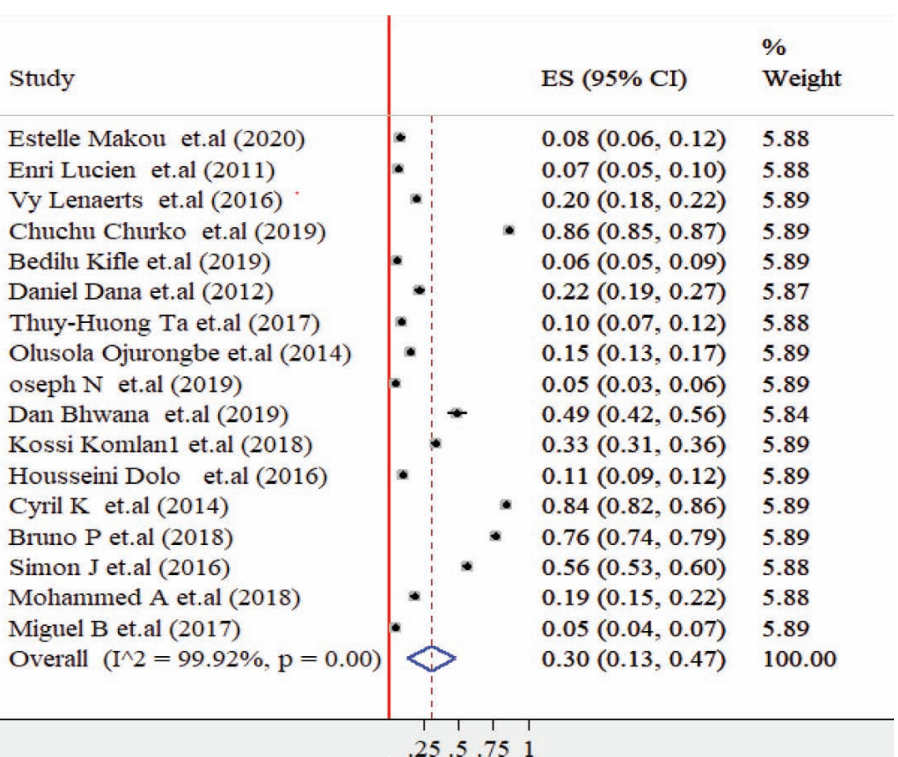

Figure 2: Forest plot of the pooled prevalence of onchocerciasis infection in sub Saharan Africa countries, $2021(n=17)$.

\section{Prevalence of Onchocerciasis Infection in sub-Saharan Africa Countries}

The pooled prevalence of onchocerciasis infection among peoples of all age groups in sub Saharan Africa countries was found to be $30 \%$ (95\% CI: 13, 47), as shown in the forest plot. Substantial heterogeneity was, however, discovered in studies ( $\mathrm{I} 2=99.92 \%, p<0.001)$. By considering this fact, we performed a random effect analysis (Figure 2).

\section{Publication bias}

The existence of publication bias was determined within the included studies. In this systematic review and meta-analysis, the funnel plot was assessed for asymmetry distribution of prevalence of onchocerciasis by visual inspection. This shows that all the studies' effect sizes were normally distributed around the center of a funnel plot due to the absence of publication bias. As defined subjectively below in the funnel map, each study's scatter plot is more clustered near zero, suggesting that there was no publication bias (Figure 3).

Besides, the publication bias was objectively assessed using Begg's and Egger's tests to rule out test of the null hypothesis: no small-study effects. The estimated bias coefficient (intercept) was 1.68 with a standard error of 1.3, giving a $p$-value of 0.21 . Using Egger's regression test with a $\mathrm{p}$-value of 0.21 tests provides strong evidence for the absence of smallstudy effects (no publication bias). Lastly, as the $p$-value is $>0.05$, was no statistical evidence of publication bias using the Begg's test for estimating the prevalence of onchocerciasis infection in Africa countries $(p=0.23)$ and $(p=0.21)$ respectively (Figure 4$)$.

\section{Subgroup analysis}

There was considerable heterogeneity identified across 17 included studies in this systematic review and meta-analysis $\left(\left(\mathrm{I}^{2}=99.92 \%, p<0.001\right)\right)$. Thus, a subgroup analysis was conducted through stratification using the variables such as the samples size, type of occupation and year of publication to Figure out the sources of heterogeneity for the pooled prevalence of onchocerciasis infection. In this systematic reviews and meta-analysis, the prevalence of onchocerciasis infection was found to be higher in some groups; however, it no significantly varied 39\% (95\% CI: 15,63$)$ in studies conducted with sample size more than $600,17 \%$ (95\% CI: 10, 23) in studies conducted with sample size of less than 600 as compared with their counterpart (Figure 5). 


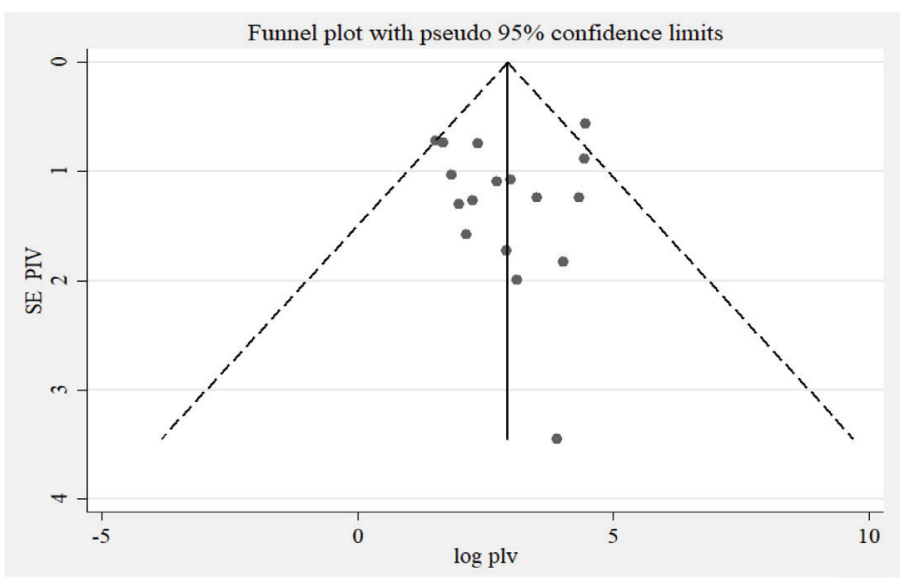

Figure 3: Funnel plot with $95 \%$ confidence limits of the prevalence of onchocerciasis infection in Africa countries, 2021 ( $n=17)$.

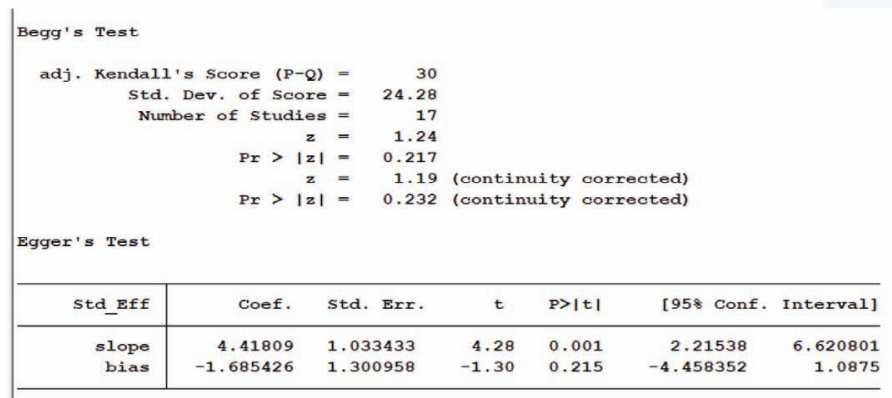

Figure 4: Begg's and Egger's test for detection of publication bias for studies included to estimated pooled prevalence of onchocerciasis infection in sub Saharan Africa countries, $2021(n=17)$.
Besides, based on the type of occupation of participants, we performed subgroup analysis. The highest prevalence of onchocerciasis infection was reported from studies involved farmers $35 \%$ (95\% CI: 12, 58) (Figure 6). Furthermore, we conducted a subgroup analysis based on the year of publication. The highest prevalence of onchocerciasis infection was reported in studies done before the year of 2015 was found to be $32 \%$ (95\% CI: 9, 56) (Figure 7).

\section{Meta-regression}

Despite the fact that the meta-regression for the 17 included studies was also performed to classify causes for heterogeneity in addition to subgroup analysis, there was no statistical meaning of significance from the meta-regression model to classify the possible source of heterogeneity (Figure 8).

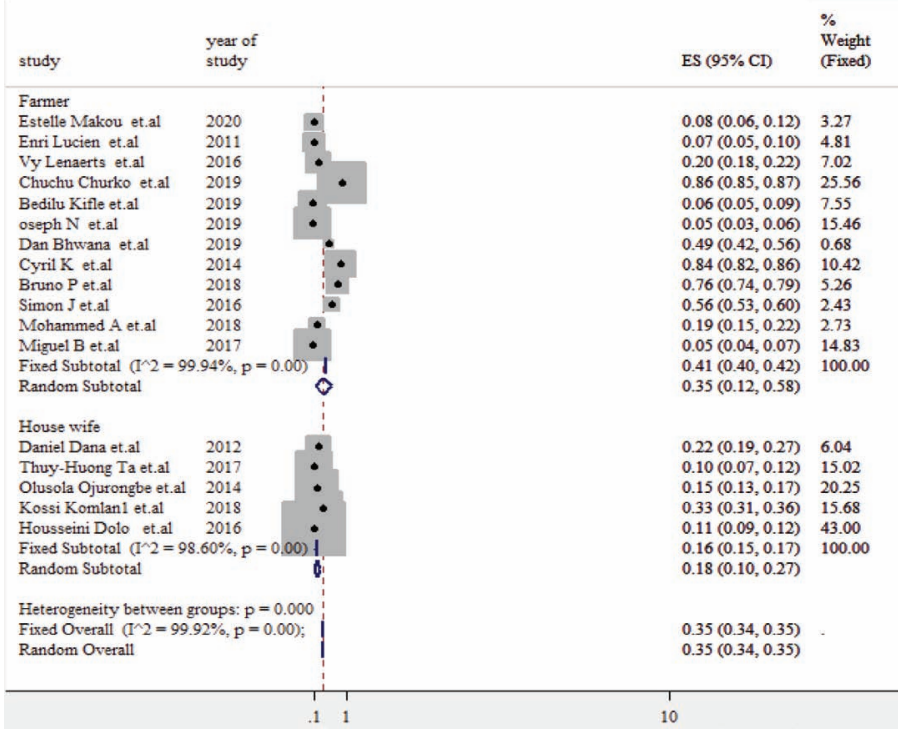

Figure 6: Subgroup analysis of prevalence of onchocerciasis infection by the type of occupation in sub-Saharan Africa countries, $2021(n=17)$.

\begin{tabular}{|c|c|c|c|}
\hline study & $\begin{array}{l}\text { year of } \\
\text { study }\end{array}$ & ES $(95 \%$ Cl) & $\begin{array}{l}\% \\
\text { Weight } \\
\text { (Fixed) }\end{array}$ \\
\hline \multicolumn{4}{|l|}{ After 2015} \\
\hline Estelle Makou et.al & 2020 & $0.08(0.06,0.12)$ & 3.85 \\
\hline Chuchu Churko et.al & 2019 & $0.86(0.85,0.87)$ & 30.16 \\
\hline Bedilu Kifle et.al & 2019 & $0.06(0.05,0.09)$ & 8.91 \\
\hline Thuy-Huong Ta et.al & 2017 & $0.10(0.07,0.12)$ & 5.99 \\
\hline oseph $\mathrm{N}$ et.al & 2019 & $0.05(0.03,0.06)$ & 18.25 \\
\hline Kossi Komlan1 et.al & 2018 & $0.33(0.31,0.36)$ & 6.25 \\
\hline Housseini Dolo et.al & 2016 & $0.11(0.09,0.12)$ & 17.15 \\
\hline Bruno P et al & 2018 & $0.76(0.74,0.79)$ & 6.21 \\
\hline Mohammed A et.al & 2018 & $0.19(0.15,0.22)$ & 3.22 \\
\hline \multicolumn{2}{|c|}{ Fixed Subtotal $\left(\mathbb{I}^{\prime \prime 2}=99.94 \%, \mathrm{p}=0.00\right)$} & $0.38(0.37,0.38)$ & 100.00 \\
\hline \multicolumn{2}{|l|}{ Random Subtotal } & $0.28(0.02,0.54)$ & \\
\hline \multicolumn{4}{|l|}{ Before 2015} \\
\hline Enri Lucien et.al & 2011 & $0.07(0.05,0.10)$ & 9.81 \\
\hline Vy Lenaerts et.al & 2016 & $0.20(0.18,0.22)$ & 14.31 \\
\hline Daniel Dana et.al & 2012 & $0.22(0.19,0.27)$ & 4.16 \\
\hline Olusola Ojurongbe et.al & 2014 & $0.15(0.13,0.17)$ & 13.95 \\
\hline Dan Bhwana et.al & 2019 & $0.49(0.42,0.56)$ & 1.39 \\
\hline Cyril K et.al & 2014 & $0.84(0.82,0.86)$ & 21.23 \\
\hline Simon J et.al & 2016 & $0.56(0.53,0.60)$ & 4.94 \\
\hline \multirow{2}{*}{\multicolumn{2}{|c|}{$\begin{array}{l}\text { Miguel b et.al } \\
\text { Fixed Subtotal }(1 \wedge 2=99.88 \%, p=0.00)\end{array}$}} & $0.05(0.04,0.07)$ & 30.22 \\
\hline & & $0.30(0.29,0.30)$ & 100.00 \\
\hline \multicolumn{2}{|l|}{ Random Subtotal } & $0.32(0.09,0.56)$ & \\
\hline \multicolumn{4}{|c|}{ Heterogeneity between groups: $p=0.000$} \\
\hline \multirow{2}{*}{\multicolumn{2}{|c|}{ Fixed Overall $\left(I^{\prime} 2=99.92 \%, p=0.00\right)$}} & $0.35(0.34,0.35)$ & \\
\hline & Random Overall & $0.35(0.34,0.35)$ & \\
\hline & & & \\
\hline
\end{tabular}

Figure 7: Sub-group analysis of prevalence of onchocerciasis infection by the year of publication in sub-Saharan Africa countries, $2021(n=17)$.
Figure 5: Subgroup analysis of the pooled prevalence of onchocerciasis infection by the sample size in sub-Saharan Africa countries, $2021(n=17)$.

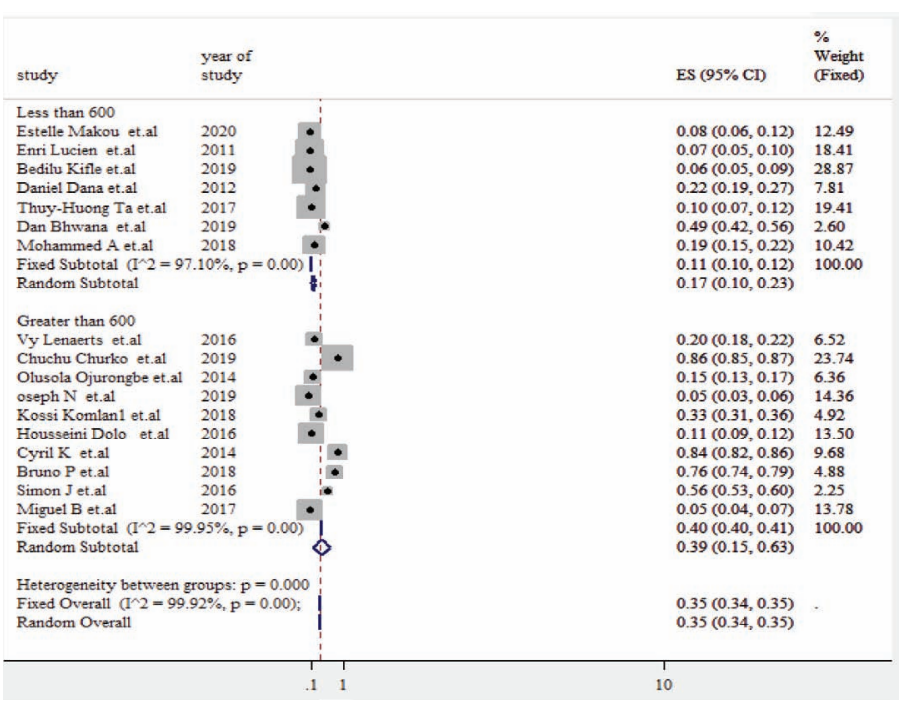




\section{Sensitivity analysis}

In this systematic review and meta-analysis, a sensitivity analysis was done to see the effect of individual studies on the pooled prevalence of occupational injury using the random-effects model. The result of the sensitivity analysis shows no single study influenced (no outlier studies) the pooled estimated prevalence of onchocerciasis. This could be due to none of the single studies being influential. The estimate was not away from each corresponding article either from its corresponding lower confidence interval or an upper confidence interval (Figure 9).

In this systematic review and meta-analysis, it was observed that the amount of variation between studies were absent (Figure 10).

\section{DISCUSSION}

The availability of concrete evidence is more relevant for policymakers, neglected tropical disease prevention partners, and other implementers. Although the mass drug administration with ivermectin drug seems to

\begin{tabular}{|c|c|c|c|c|c|c|c|}
\hline Meta-regression & & & & Number & obs & $=$ & 17 \\
\hline REML estimate of be & en-study v & riance & & tau2 & & & 2476 \\
\hline 8 residual variatio & Le to hete & ogeneity & & I-squar & res & $=$ & .008 \\
\hline Proportion of betwe & study vari & nce explai & & Adj $\mathrm{R}-\mathrm{s}$ & ared & $=57$ & .488 \\
\hline Joint test for all & ariates & & & Model $\mathrm{F}$ & & $=$ & 1.04 \\
\hline With Knapp-Hartung & ification & & & Prob $>$ & & $=0$. & 4888 \\
\hline logplv & $\exp (b)$ & Std. Err. & $\mathrm{t}$ & $\mathrm{P}>|\mathrm{t}|$ & {$[958$} & Conf. & Interval] \\
\hline greaterthan15years & .3780759 & 1.607472 & -0.23 & 0.826 & .000 & 0163 & 8787.227 \\
\hline lessthan15years & .0716527 & .1992606 & -0.95 & 0.375 & .000 & 0999 & 51.41396 \\
\hline below600 & .1207265 & .2480276 & -1.03 & 0.338 & .000 & 9375 & 15.54591 \\
\hline above 600 & 2.024095 & 4.676204 & 0.31 & 0.769 & .008 & 55845 & 477.25 \\
\hline after 2015 & 2.252792 & 6.471944 & 0.28 & 0.786 & .002 & 5262 & 2009.001 \\
\hline before2015 & 1.600004 & 7.083987 & 0.11 & 0.918 & .000 & 0454 & 56349.71 \\
\hline male & .659959 & 2.759591 & -0.10 & 0.924 & .000 & 0335 & 12990.84 \\
\hline female & .5271335 & .637327 & -0.53 & 0.613 & .030 & 2205 & 9.194732 \\
\hline farmer & 1.533977 & 2.053683 & 0.32 & 0.759 & .064 & 17069 & 36.36527 \\
\hline _cons & 65.40988 & 200.552 & 1.36 & 0.215 & .046 & 4463 & 92116.1 \\
\hline
\end{tabular}

Figure 8: Meta-regression of the 17 included studies to estimate the pooled prevalence of onchocerciasis infection in sub-Saharan Africa countries, 2021 $(n=17)$.

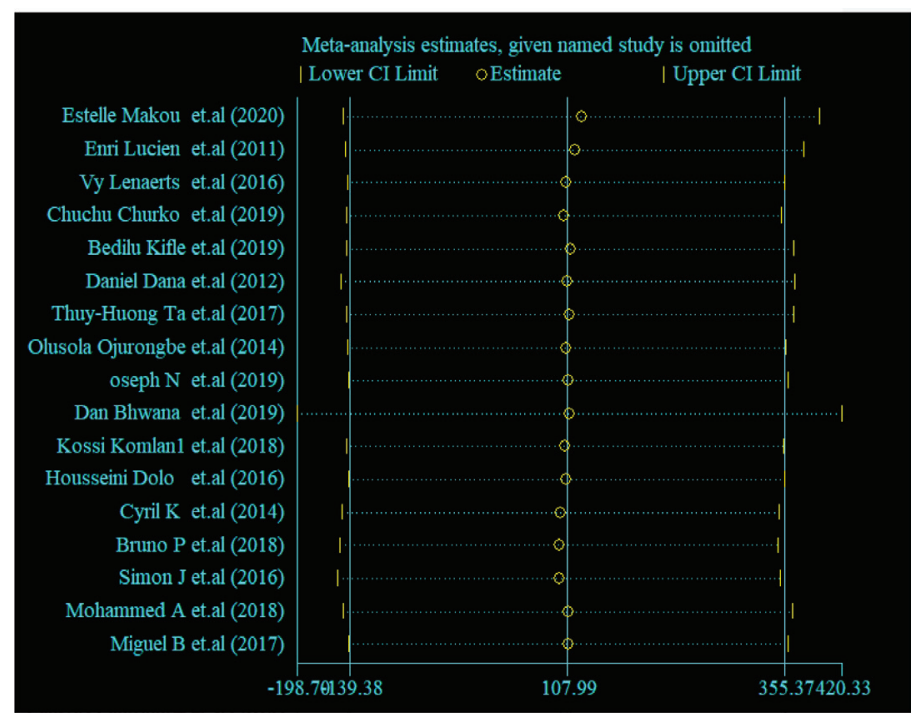

Figure 9: Sensitivity of this systematic review and meta-analysis of the 20 included studies to estimate the pooled prevalence of onchocerciasis infection in sub-Saharan Africa countries, 2021 ( $n=17)$.

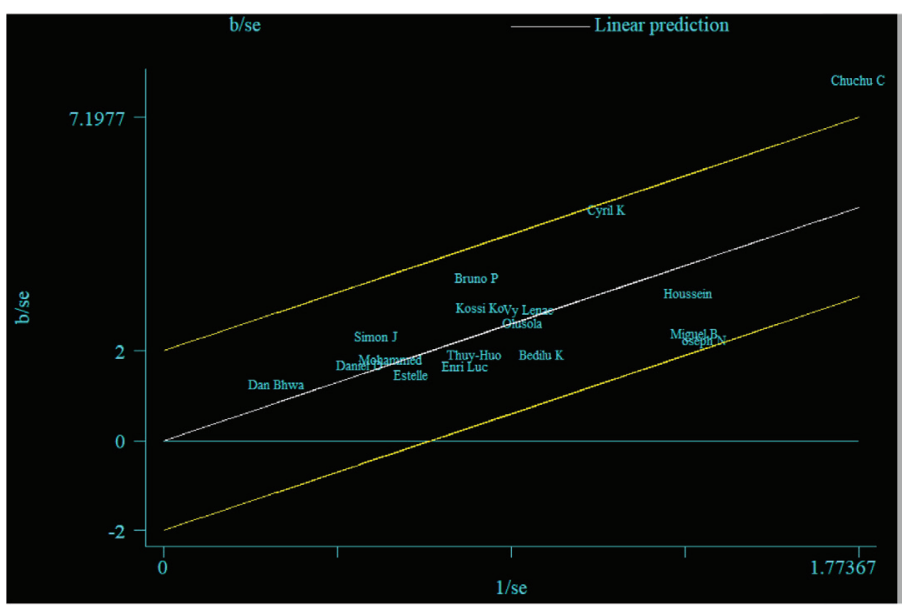

Figure 10: Galbraith plot showing provides a graphical display of the amount of heterogeneity from a meta-analysis.

reduce microfilaria prevalence in the communities effectively, the present review result showed that the pooled prevalence of onchocerciasis infection in sub-Saharan Africa countries was very high.

According to the present review finding, the burdens of onchocerciasis infection in sub-Saharan African countries might negatively impact they hope to achieve the world health organization target for eliminating onchocerciasis by $2025 . .^{50}$ The finding of this systematic review and meta-analysis is much higher than other findings reported from different parts of the world. It is higher than review reports from Switzerland, ${ }^{51}$ France, ${ }^{52,53}$ Canada, ${ }^{54}$ Spain, ${ }^{55}$ Nigeria,${ }^{56}$ Cameroon.${ }^{57}$ However, the pooled prevalence of onchocerciasis was lower than the study from Brazilian Amazon, ${ }^{58}$ and Burkina Faso. ${ }^{29}$

Methodological variation in estimating the outcome of the analysis and variation in geographical location, the study period, and socioeconomic status of the study participants may be credited with the potential reason for the above difference. The majority of the previous studies were done only in few countries. However, the present review comprehensively incorporated a vast geographical location. The disparity may also be rationalized by the difference in compliance with the neglected tropical disease prevention program, and the inadequate implementation of recommended intervention may be the potential explanation for this difference. Besides, the possible explanation for this difference is that onchocerciasis infection occurs in developing countries with the most significant population living in resource-limited settings and poor sanitation. According to this study, the local government gives minimal attention to the implementation of comprehensive neglected tropical disease control programs stated by various actors to avoid exposure to onchocercal volvulus. There are likely to be limited onchocerciasis surveillance systems.

The subgroup analysis of this systematic review and meta-analysis revealed that the prevalence of onchocerciasis was varied across the sample size, type of occupation, and year of publication. Based on the sample size sub-group analysis, the prevalence of onchocerciasis was higher in studies with a sample size of more than 600 than studies with a sample size of less than 600 . However, it was not significantly varying. Moreover, in this systematic review and meta-analysis, the highest prevalence of onchocerciasis infection was reported in studies conducted before the 2015 year compared to the survey report conducted after the year 2015 in the sub-group analysis. The result was inconsistent with a systematic study of the research from Global, and regional Figures, ${ }^{59}$ Saudi Arabia, ${ }^{60}$ Nigeria, ${ }^{61}$ Venezuelan Amazonian, ${ }^{62}$ The possible 
explanation for these variations might be related to the inadequate implementation of disease-specific intervention strategies before 2015 . Besides, it might be due to the local government and stakeholder's commitment being minimal compared to the present era.

Furthermore, the results of the current review from the subgroup analysis by the type of occupation in sub-Saharan Africa countries: relatively the highest prevalence of onchocerciasis infection was reported among farmers compared to housewives. Thus, we understood that the burdens of onchocerciasis infection were varied by the type of occupation, which needs a further explanation for this variation. Therefore, to reduce this high magnitude and its future associated economic cost of onchocerciasis infection, the local government and other concerned bodies should consider the implementation, progress, and effectiveness of WHO's prevention and control strategies designed for neglected tropical diseases. Based on the present findings, the nature of peoples exposed to onchocercal volvulus depends on the populations' occupation and socioeconomic status. A small segment of population coverage and significant underreporting of onchocerciasis infection and disorganized documentation, and inadequate documentation of all data system forms are the key factors contributing to underestimating. There is a lack of a robust national surveillance and notification framework for onchocerciasis infection in many African countries. This means that the number of onchocerciasis infections is underreported. As a result, underreporting of onchocerciasis infection limits our ability to diagnose and resolve associated health problems. New programs, models, and approaches must be created to identify the causes of underreporting onchocerciasis infection in Africa.

\section{Strengths and limitations}

This research review analyzed evidence from primary studies performed in sub-Saharan African countries in compliance with PRISMA guidelines. One of this review's strengths is that it covers a large geographic region. Our search was carried out in close collaboration with a specialist research librarian, with three researchers screening and extracting data using a standardized extraction form. In the selection process, the inter-rater agreement between reviewers was statistically evaluated. Furthermore, this systematic analysis and meta-analyses were the first of their kind on onchocerciasis infection in sub-Saharan African countries. This review, like other systematic reviews and meta-analyses, has its own set of limitations. As a result, most of the studies in this study were crosssectional, and other confounding variables may influence the dependent variable. Furthermore, the researchers conducted in other parts of African countries were not included in this systematic study and meta-analysis. The heterogeneity of the studies made it difficult to pool information and deliver reliable proof. Many analyses only included a few reports, lowering the intensity of the proof. Finally, although the identification of articles published in languages other than English was compatible with others, we only looked at articles published in English.

\section{CONCLUSION AND RECOMMENDATION}

The results from this systematic review and meta-analysis demonstrated that onchocerciasis infection remains an immense public health concern in Sub-Saharan Africa despite effective intervention. This high pooled prevalence of onchocerciasis infection needs immediate action and special considerations.

Hence, the local government and other stakeholders should be followed and implement rigorous and comprehensive onchocerciasis prevention strategies such as improved sanitation, vector control, and mass drug administration campaigns, and multifaceted methods should be the areas of action, according to our recommendations. Besides, integrated epidemiological surveillance of onchocerciasis should be warranted to review the implementation of neglected tropical disease prevention packages and interventions in the region. Future research and development towards new strategies and interventions against the disease should be crucial.

\section{ACKNOWLEDGEMENT}

The authors thank the primary authors for their contribution to this review.

\section{Ethics approval and consent to participate}

No ethical approval was necessary because data was collected and analyzed for previously reported research in which primary investigators was obtain informed consent.

\section{Consent for publication}

For publication in a peer-reviewed journal, we were preparing a manuscript and present the findings at conferences.

\section{Availability of data and materials}

The datasets used and/or analyzed during the current study are available from the corresponding author on reasonable request.

\section{CONFLICT OF INTEREST}

The authors declare no conflict of interest.

\section{Author Contributions}

Conceptualization: Mitiku Bonsa Debela

Data curation: Mitiku Bonsa Debela, Dejene Hailu Kassa and Taklu Marama Mokonnon

Formal analysis: Mitiku Bonsa Debela, Dejene Hailu Kassa and Taklu Marama Mokonnon

Investigation: Mitiku Bonsa Debela, Dejene Hailu Kassa and Taklu Marama Mokonnon

Methodology: Mitiku Bonsa Debela, Dejene Hailu Kassa

Project administration: Mitiku Bonsa Debela, and Dejene Hailu Kassa

Resources: Mitiku Bonsa Debela, and Dejene Hailu Kassa

Software: Mitiku Bonsa Debela, Dejene Hailu Kassa, and Taklu Marama Mokonnon

Supervision: Dejene Hailu Kassa, and Taklu Marama Mokonnon

Validation: Dejene Hailu Kassa, avd Taklu Marama Mokonnon

Visualization: Mitiku Bonsa Debela, and Taklu Marama Mokonnon

Writing - original draft: Mitiku Bonsa Debela and Taklu Marama Mokonnon

Writing - review \& editing: Mitiku Bonsa Debela, Dejene Hailu Kassa, and Taklu Marama Mokonnon

\section{REFERENCES}

1. Mitra AK, Mawson AR. Neglected tropical diseases: epidemiology and global burden. Trop Med Infect Dis. 2017;2(3). doi: 10.3390/tropicalmed2030036, PMID 30270893.

2. WHO. Ending Neglected Tropical Diseases: A gateway to Universal Health Coverage: fifth progress report on the London Declaration on NTDs; 2017 [cited 19/7/2021]. Available from: https://www.who.int/docs/default-source/ documents/evaluation/evaluation-ntd-report.pdf.

3. Hotez PJ, Molyneux DH, Fenwick A, Kumaresan J, Sachs SE, Sachs JD Savioli L. Control of neglected tropical diseases. N Engl J Med. 2007;357(10):1018-27. doi: 10.1056/NEJMra064142, PMID 17804846.

4. Hotez PJ. Mass drug administration and integrated control for the world's highprevalence neglected tropical diseases. Clin Pharmacol Ther. 2009;85(6):659-64. doi: 10.1038/clpt.2009.16, PMID 19322166.

5. Murdoch ME. Mapping the burden of onchocercal skin disease. Br J Dermatol. 
2021;184(2):199-207. doi: 10.1111/bjd.19143, PMID 32302410.

6. WHO. Accelerating work to overcome the global impact of neglected tropical diseases: A road map for implementation. p. 1-42; 2012 [cited 19/7/2021] Available from: https://apps.who.int/iris/handle/10665/70809.

7. WHO. 681 Progress report on the elimination of human onchocerciasis, 2016-2017. Wkly Epidemiol Rec. 2017;92(45):681-94. PMID 29130679.

8. Vos T, Barber RM, Bell B, Bertozzi-Villa A, Biryukov S, Bolliger I, et al. Global, regional, and national incidence, prevalence, and years lived with disability for 301 acute and chronic diseases and injuries in 188 countries, 1990-2013: a systematic analysis for the Global Burden of Disease Study 2013. Lancet. 2015:386(9995):743-800. doi: 10.1016/S0140-6736(15)60692-4.

9. Herricks JR, Hotez PJ, Wanga V, Coffeng LE, Haagsma JA, Basáñez MG, Buckle G, Budke CM, Carabin $\mathrm{H}$, Fèvre EM, Fürst $\mathrm{T}$, Halasa YA, King $\mathrm{CH}$, Murdoch ME, Ramaiah KD, Shepard DS, Stolk WA, Undurraga EA, Stanaway JD, Naghavi M, Murray CJL. The global burden of disease study 2013: what does it mean for the NTDs? PLOS Negl Trop Dis. 2017;11(8):e0005424. doi: 10.1371/journal. pntd.0005424, PMID 28771480.

10. GBD. Global, regional, and national incidence, prevalence, and years lived with disability for 328 diseases and injuries for 195 countries, 1990-2016: a systematic analysis for the Global Burden of Disease Study 2016. Lancet. 2017;390(10100):1211-59. doi: 10.1016/S0140-6736(17)32154-2, PMID 28919117.

11. WHO. Onchocerciasis; 2019 [cited 19/7/2021]. Available from: https://www. who.int/news-room/fact-sheets/detail/onchocerciasis.

12. WHO. Progress report on the elimination of human onchocerciasis. Wkly Epidemiol Rec. 2019;94:513-24.

13. Lakwo T, Oguttu D, Ukety T, Post R, Bakajika D. Onchocerciasis elimination: progress and challenges. Res Rep Trop Med. 2020;11:81-95. doi: 10.2147/ RRTM.S224364, PMID 33117052.

14. Mark T, Achim H, Moses B. Lymphatic filariasis and onchocerciasis. PubMed Lancet. 2010;376(9747):1175-85. doi: 10.1016/s0140-6736(10)60586-7.

15. Noma M, Zouré HG, Tekle AH, Enyong PA, Nwoke BE, Remme JH. The geographic distribution of onchocerciasis in the 20 participating countries of the African Programme for Onchocerciasis Control: (1) priority areas for ivermectin treatment. Parasit Vectors. 2014;7:325. doi: 10.1186/1756-3305-7-325, PMID 25053266

16. Aiah G, John C, Peter T. Prevalence of onchocerciasis (river-blindness) in Selected Villages of the Gorama Chiefdom, Sierra Leone Tropenmedizin PubMed 2014. doi: 10.1016/s0033-3506(86)80097-x.

17. Michel M, Patrick S, Floribert T. Onchocerca volvulus as a risk factor for developing epilepsy in onchocerciasis endemic regions in the Democratic Republic of the Congo: a case control study. Vol. 79. BMC; 2018. p. 7.

18. Mweya CN, Kalinga AK, Kabula B, Malley KD, Ruhiso MH, Maegga BT. Onchocerciasis situation in the Tukuyu focus of southwest Tanzania after ten years of ivermectin mass treatment. Tanzan Health Res Bull. 2007;9(3):174-9. doi: 10.4314/thrb.v9i3.14325, PMID 18087895.

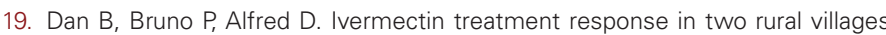
with a high prevalence of onchocerciasis and epilepsy, Mahenge, Tanzania. University of Antwerp, Global Health Institute 2019. p. 1-3. doi: 10.21203/ rs.3.rs-118303/v1.

Mahdy MAK, Abdul-Ghani R, Abdulrahman TAA, Al-Eryani SMA, Al-Mekhlafi AM, Alhaidari SAA, Azazy AA. Onchocerca volvulus infection in Tihama region - west of Yemen: continuing transmission in ivermectin-targeted endemic foci and unveiled endemicity in districts with previously unknown status. PLOS NegITrop Dis. 2018;12(3):e0006329. doi: 10.1371/journal.pntd.0006329, PMID 29505580.

20. Gebremedhin G, Zeleke M, Delenasaw Y. Status of parasitological indicators and morbidity burden of onchocerciasis after years of successive implementation of mass distribution of ivermectin in selected communities of Yeki and Asosa districts. Available from: https://bmcpublichealth.biomedcentral.com/ articles/10.1186/s12889-020-09344-7. Vol. 20. BMC. Ethiopia; 2020. p. 1-12.

21. Taye A, Gebre-Michael T, Taticheff S. Onchocerciasis in Gilgel Ghibe River Valley southwest Ethiopia. East Afr Med J. 2000;77(2):116-20. doi: 10.4314/ eamj.v77i2.46411, PMID 10774086.

22. Kifle B, Woldemichael K, Nigatu M. Prevalence of Onchocerciasis and Associated Factors among Adults Aged $\geq 15$ Years in Semen Bench District, Bench Maji Zone, Southwest Ethiopia: Community Based Cross-Sectional Study. Adv Public Health. 2019;2019:1-9. doi: 10.1155/2019/7276230.

23. Otabil KB, Gyasi SF, Awuah E, Obeng-Ofori D, Atta-Nyarko RJ, Andoh D, Conduah B, Agbenyikey L, Aseidu P, Ankrah CB, Nuhu AR, Schallig HDFH. Prevalence of onchocerciasis and associated clinical manifestations in selected hypo endemic communities in Ghana following long-term administration of ivermectin. BMC Infect Dis. 2019;19(1):431. doi: 10.1186/s12879-019-4076-2, PMID 31101085

24. David O, Edson B, Charles R, Peace H. Onchocerciasis serosurveillance in Uganda American Society of Tropical Medicine and Hygiene 2013:1-34 [cited 19/7/2021]. Available from: https://www.ncbi.nlm.nih.gov/pmc/articles/ PMC3919245/\#.

25. Aza'ah RA, Sumo L, Ntonifor NH, Bopda J, Bamou RH, Nana-Djeunga HC. Point prevalence mapping reveals hotspot for onchocerciasis transmission in the Ndikinimeki Health District, Centre Region, Cameroon. Parasit Vectors. 2020;13(1):519. doi: 10.1186/s13071-020-04387-6, PMID 33066826
Tsapi EM, Todjom FG, Gamago GA, Pone JW, Teukeng FFD. Prevalence of onchocerciasis after seven years of continuous community-directed treatment with ivermectin in the Ntui health district, Centre region, Cameroon. Pan Afr Med J. 2020;36:180. doi: 10.11604/pamj.2020.36.180.20765, PMID 32952824.

26. Katabarwa MN, Eyamba A, Nwane P, Enyong P, Kamgno J, Kueté T, Yaya $S$, Aboutou R, Mukenge L, Kafando C, Siaka C, Mkpouwoueiko S, Ngangue D, Biholong BD, Andze GO. Fifteen years of annual mass treatment of onchocerciasis with ivermectin have not interrupted transmission in the west region of Cameroon. J Parasitol Res. 2013;2013:420928. doi: 10.1155/2013/420928, PMID 23691275.

27. Nikièmaa AS, Koalaa L, Postb RJ. Paré A. CM: onchocerciasis prevalence, human migration and risks for onchocerciasis elimination in the Upper Mouhoun Nakambe and Nazinon river basins in Burkina Faso. BMC; 2018.

28. Hendy A, Krüger A, Pfarr K, De Witte J, Kibweja A, Mwingira U, Dujardin JC Post R, Colebunders R, O'Neill S, Kalinga A. The blackfly vectors and transmission of Onchocerca volvulus in Mahenge, south eastern Tanzania. Acta Trop. 2018;181:50-9. doi: 10.1016/j.actatropica.2018.01.009, PMID 29410302.

29. CIA. Fertility rate, total (births per woman). Data. 2016.

30. Janneh A. General debate on national experience in population matters: adolescents and youth. United Nations Economic Commission for Africa 2015 [cited 19/7/2021]. Available from: https://www.un.org/en/development/desa/population/pdf/commission/2012/country/Agenda\%20item\%208/Decisions\%20 and\%20resolution/Resolution\%202012_1_Adolescents\%20and\%20Youth.pdf.

31. United Nations Department of Economic and Social Affairs. PD: overall total population; 2019.

32. White A. Senegal: birth of a new tobacco control group. Tob Control. 1999;8(1):16-7. doi: 10.1136/tc.8.1.14-d, PMID 10465810.

33. Elizabeth $\mathrm{H}$. Health professionals, population density, and adequate antenatal care in nine Sub-Saharan African countries 2019:1-5.

34. Kaiser C, Mmbando BP, Siewe Fodjo JN, Suykerbuyk P, Mnacho M, Kakorozya A, Matuja W. Hendy A, Greter H, Makunde WH, Colebunders R. Onchocerciasisassociated epilepsy: another piece in the puzzle from the Mahenge mountains, southern Tanzania. Infect Dis Pover. 2019;8(1):35. doi: 10.1186/s40249-0190545-5, PMID 31122275.

35. Chuchu C, Manaye Y, Abinet T. Ivermectin treatment coverage validation in two onchocerciasis endemic districts in Ethiopia: A community-based cross-sectional study. J Multidiscipline Healthc. 2019;14:1-41. doi: 10.2147/jmdh.s288239.

36. Dan B, Bruno P. Ivermectin treatment response in two rural villages with a high prevalence of onchocerciasis and epilepsy. Mahenge, Tanzania; 2019. doi: 10.21203/rs.3.rs-118303/v1.

37. Dana D, Debalke S, Mekonnen Z, Kassahun W, Suleman S, Getahun K, Yewhalaw D. A community-based cross-sectional study of the epidemiology of onchocerciasis in unmapped villages for community directed treatment with ivermectin in Jimma Zone, southwestern Ethiopia. BMC Public Health 2015;15:595. doi: 10.1186/s12889-015-1888-x, PMID 26130117.

38. Lenaerts E, Mandro M, Mukendi D, Suykerbuyk P, Dolo H, Wonya'Rossi D, Ngave F, Ensoy-Musoro C, Laudisoit A, Hotterbeekx A, Colebunders R. High prevalence of epilepsy in onchocerciasis endemic health areas in Democratic Republic of the Congo. Infect Dis Poverty. 2018;7(1):68. doi: 10.1186/s40249018-0452-1. PMID 30064504.

39. Henri L, Dickson Sh, Jules C. Prevalence of onchocerciasis in the Fundong Health District, Cameroon after 6 years of continuous community-directed treatment with ivermectin. Pan Afr Med J. 2011:1-12

40. Dolo H, Coulibaly YI, Dembele B, Guindo B, Coulibaly SY, Dicko I, Doumbia SS, Dembele M, Traore MO, Goita S, Dolo M, Soumaoro L, Coulibaly ME, Diallo AA, Diarra D, Zhang Y, Colebunders R, Nutman TB. Integrated seroprevalence-based assessment of Wuchereria bancrofti and Onchocerca volvulus in two lymphatic filariasis evaluation units of Mali with the SD Bioline onchocerciasis/LF IgG4 Rapid Test. PLOS Negl Trop Dis. 2019;13(1):e0007064. doi: 10.1371/journal. pntd.0007064, PMID 30699120

41. Siewe JNF, Ukaga CN, Nwazor EO, Nwoke MO, Nwokeji MC, Onuoha BC, Nwanjor SO, Okeke J, Osahor K, Chimechefulam L, Ogomaka Al, Amaechi AA Ezenwa Cl, Ezike MN, Ikpeama C, Nwachukwu O, Eriama-Joseph Al, Nwoke BEB, Colebunders R. Low prevalence of epilepsy and onchocerciasis after more than 20 years of ivermectin treatment in the Imo River Basin in Nigeria. Infect Dis Poverty. 2019;8(1):8. doi: 10.1186/s40249-019-0517-9, PMID 30670093.

42. Komlan K, Vossberg PS, Gantin RG, Solim T, Korbmacher F, Banla M, Padjoudoum K, Karabou P, Köhler C, Soboslay PT. Onchocerca volvulus infection and serological prevalence, ocular onchocerciasis and parasite transmission in northern and central Togo after decades of Simulium damnosum s.l. vector control and mass drug administration of ivermectin. PLOS NegI Trop Dis. 2018;12(3):e0006312. doi: 10.1371/journal.pntd.0006312, PMID 29494606.

43. Brito M, Paulo R, Van-Dunem P, Martins A, Unnasch TR, Novak RJ, Jacob B, Stanton MC, Molyneux DH, Kelly-Hope LA. Rapid integrated clinical survey to determine prevalence and co-distribution patterns of lymphatic filariasis and onchocerciasis in a Loa loa co-endemic area: The Angolan experience. Parasite Epidemiol Control. 2017;2(3):71-84. doi: 10.1016/i.parepi.2017.05.001. PMID 29774284.

44. Ojurongbe O, Akindele AA, Adeleke MA, Oyedeji MO, Adedokun SA, Ojo JF, Akinleye CA, Bolaji OS, Adefioye OA, Adeyeba OA. Co-endemicity of loiasis 
and onchocerciasis in rain forest communities in Southwestern Nigeria. PLOS Negl Trop Dis. 2015;9(3):e0003633. doi: 10.1371/journal.pntd.0003633, PMID 25812086

45. O'Hanlon SJ, Slater HC, Cheke RA, Boatin BA, Coffeng LE, Pion SD, Boussinesq M, Zouré HG, Stolk WA, Basáñez MG. Model-based geostatistical mapping of the prevalence of Onchocerca volvulus in west Africa. PLOS Negl Trop Dis. 2016;10(1):e0004328. doi: 10.1371/journal.pntd.0004328, PMID 26771545.

46. Ta TH, Moya L, Nguema J, Aparicio P, Miguel-Oteo M, Cenzual G, Canorea I, Lanza M, Benito A, Crainey JL, Rubio JM. Geographical distribution and species identification of human filariasis and onchocerciasis in Bioko Island, Equatorial Guinea. Acta Trop. 2018;180:12-7. doi: 10.1016/j.actatropica.2017.12.030. PMID 29289559.

47. Murdoch ME. Onchodermatitis. Curr Opin Infect Dis. 2010;23(2):124-31. doi: 10.1097/QCO.0b013e328336a256. PMID 20071985.

48. Kuesel AC. Research for new drugs for elimination of onchocerciasis in Africa. Int J Parasitol Drugs Drug Resist. 2016;6(3):272-86. doi: 10.1016/j.ijpddr.2016.04.002, PMID 27693536.

49. Kappagoda S, loannidis JP. Prevention and control of neglected tropical diseases: overview of randomized trials, systematic reviews and meta-analyses. Bull World Health Organ. 2014;92(5):356-366C. doi: 10.2471/BLT.13.129601, PMID 24839325.

50. Kaiser C, Pion SD, Boussinesq Mbastien D, Boussinesq M et al. Case-control studies on the relationship between onchocerciasis and epilepsy: systematic review and meta-analysis. PLOS Negl Trop Dis. 2013;7(3):e2147. doi: 10.1371/ journal.pntd.0002147, PMID 23556028.

51. Se'bastien D, Christophe K, amandine C. Epilepsy in onchocerciasis Endemic Areas: systematic Review and meta-analysis of population-based surveys. PLOS Negl Trop Dis. 2010;3(6):1-12.

52. Puente S, Ramirez-Olivencia G, Lago M, Subirats M, Perez-Blazquez E, Bru F, Garate T, Vicente B, Belhassen-Garcia M, Muro A. Dermatological manifestations in onchocerciasis: A retrospective study of 400 imported cases. Enferm Infecc
Microbiol Clin (Engl Ed). 2018;36(10):633-9. doi: 10.1016/j.eimc.2017.11.016, PMID 29275076.

53. Charles O, Odama R. Systematic review on onchocerciasis infection in Nigeria in the past five decades. Int J Med Public Health. 2020;10(1). doi: 10.5530/ ijmedph.2020.1.1.

54. Nana-Djeunga HC, Domche A, Niamsi-Emalio $Y$, Moungui HC, Walker $M$, Basáñez MG, Kamgno J. Situation analysis of onchocerciasis in Cameroon: a protocol for systematic review of epidemiological studies and impact of disease control interventions. Syst Rev. 2020;9(1):27. doi: 10.1186/s13643-020-1287-y, PMID 32046791.

55. Guilherme H, Karen J, Van W. Short report: ocular onchocerciasis in the Yanomami communities from Brazilian amazon: effects on intraocular pressure. Vol. 1. American Society of Tropical Medicine and Hygiene; 2014. p. 90.

56. GBD 2017 Disease and Injury Incidence and Prevalence Collaborators. Global regional, and national incidence, prevalence, and years lived with disability for 354 diseases and injuries for 195 countries and territories, 1990-2017: a systematic analysis for the Global Burden of Disease Study 2017. Lancet. 2018;392(10159):1789-858. doi: 10.1016/S0140-6736(18)32279-7, PMID 30496104.

57. Cheema AH, El-Bihari S, Ashour NA, Ali AS. Onchocerciasis in camels (Camelus dromedarius) in Saudi Arabia. J Helminthol. 1984;58(4):279-85. doi: 10.1017/ s0022149x0002513x. PMID 6520373.

58. Ebomoyi E, Omonisi M. Human ecology and behavior in onchocerciasis control in isolated villages of Kwara State, Nigeria. Jstor. 2012;20.

59. Carlos B, Marisela E. Néstor J. Villamizar: evidence of suppression of onchocerciasis transmission in the Venezuelan Amazonian focus. BMC; 2016. p. 40. doi: 10.1186\%2Fs13071-016-1313-z

60. CDC. Progress toward elimination of onchocerciasis in the Americas - 19932012. MMWR Morb Mortal Wkly Rep. 2013;62(20):405-8. PMID 23698606.

Cite this article : Debela MB, Kassa DH, Mokonnon TM. Prevalence of Onchocerciasis Infection in the Sub-saharan Africa Countries: A Systematic Review and Meta-analysis. Int J Med Public Health. 2021;11(4):170-8. 